\title{
Operative Management of closed fracture shaft humerus: comparative study between MIPPO anterior bridge platting Vs conventional ORIF
}

\author{
Kaushal Anand ${ }^{1}$, Abhishek Gol ${ }^{2}$, Nirav Mungalpara ${ }^{3, *}$, Sagar Usdadia $^{4}$, Rasik Shiyal ${ }^{5}$ \\ ${ }^{\mathbf{1}}$ Assistant Professor, ${ }^{2-5}$ Resident, Dept. of Orthopaedics, B.J. Medical College, Ahmedabad, Gujarat, India
}

*Corresponding Author:

Email: drnirav.mungalpara.nm@gmail.com

\begin{abstract}
Aim: Operative treatment of shaft humerus fracture continues to evolve. MIPPO is one of such emerging operative procedure which does give excellent results over conventional ORIF platting. We are comparing overall functional outcome and surgical complexity of MIPPO (Minimal Invasive Percutaneous Plate Osteosynthesis) vs conventional ORIF (Open Reduction and Internal Fixation) by posterior approach in this study.

Materials and Methods: A retrospective clinical study was done on 50 patients with shaft humerus fractures treated with MIPPO anterior bridge plating and posterior platting by trained single surgeon at a tertiary trauma care centre in the Department of Orthopaedics, BJ medical college, Civil hospital Ahmedabad between October 2016 and May 2018. Follow up data was collected and patients were called for clinical and radiological evaluation.

Results: 24 of the $25(96 \%)$ MIPPO patients and 22 of the $25(88 \%)$ ORIF patients achieved union. Average union times for the MIPPO and ORIF groups were 19.3 and 16.1 weeks respectively. At the end functional outcome of both the technique is as follows: the Mayo Elbow Performance Score (MEPS; range 95-100) were 90\% in 20 cases $(n=20)$ operated with conventional ORIF posterior plating and 20 cases operated with MIPPO plating which is excellent scores. Two cases operated with ORIF posterior plating and three cases with MIPPO plating are showing a good MEPS score.

Conclusions: Even though MIPPO is surgically demanding, fracture hematoma is not disturbed in it therefore it gives better and fast union than conventional ORIF.
\end{abstract}

Keywords: Conventional ORIF, MIPPO platting, Fracture, Humerus.

\section{Introduction}

The first description of a diaphyseal humeral fracture goes back to ancient Egypt and has been recorded on the Edwin Smith Papyrus, the world's oldest surviving surgical text that was written in Egyptian hieratic script around the $17^{\text {th }}$ century BC. The author of the papyrus described the fracture of the humerus and proposed conservative treatment. ${ }^{1}$

The most common reason for a humeral shaft fracture is a fall, followed by motor vehicle accident. ${ }^{2}$ Other causes that account for humeral shaft fractures include sporting activities, working accidents, fall from a height, violence, and bone pathology. Pathologic and open fractures of the humeral shaft are uncommon.

\section{Classification}

The system designates the humerus as a bone (1), divided into three parts: Proximal (11), diaphyseal (12), and distal (13). The diaphyseal segment is further divided into three types: AO/OTA classification consists of three types of fracture (type A-simple, type B-wedge, type C-comminuted) in term of increasing complexity of the fracture. Simple fractures (12-A) consisting of two main fragments - proximal and distal, wedge fractures (12-B) where there are one or more intermediate fragments with contact between the main fragments after reduction, and complex fractures (12-C) where there is more comminution and no contact of the main proximal and distal fragments after reduction. ${ }^{3}$
The types are further divided into three groups depending on the morphology, from "benign" (1) to "difficult" (3), and each group into three subgroups that define the proximal, middle, or distal zone of the diaphysis, where the fracture happened.

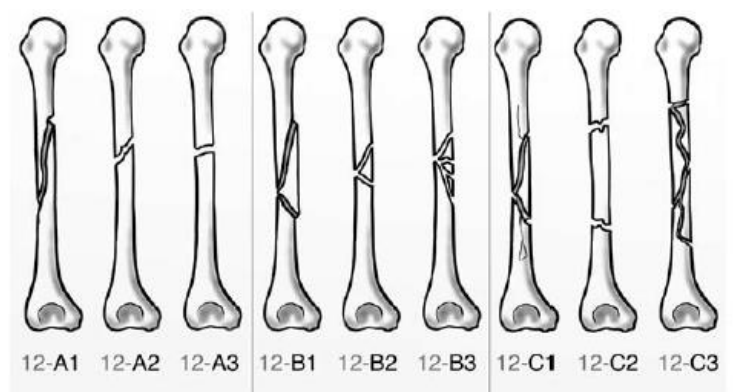

Fig. 1

Aim

To compare the overall functional outcome and surgical complexity of MIPPO (Minimal Invasive Percutaneous Plate Osteosynthesis) vs conventional ORIF (Open Reduction and Internal Fixation) by posterior plate

\section{Materials and Methods}

A retrospective clinical study was done on 50 patients with shaft humerus fractures treated with MIPPO anterior bridge plating and conventional ORIF by posterior plating by trained single surgeon at a 
tertiary trauma care center in the Department of Orthopedics, BJ medical college, Civil hospital, Ahmedabad between October 2016 and May 2018. The mean follow-up study time was approximately 13 months. A Dynamic compression plate (from Nebula Company) was used in all 50 patients.

Inclusion criteria

1. Age 20-50 years old

2. A fracture that is at least $5 \mathrm{~cm}$ distal to the surgical neck of humerus and $5 \mathrm{~cm}$ proximal to olecranon fossa

3. Gustilo-Anderson grades I or II open fracture

4. A fracture associated with poly-trauma

5. Fresh trauma within 2 week

Exclusion criteria

1. Gustilo Anderson grade 3 and 4 fracture

2. Patient with medical comorbidities

3. Pathological fracture

Operating technique

Minimally invasive percutaneous plate osteosynthesis (MIPPO)

All patients who were operated with MIPPO approach were in a supine position; with around 45 degree abduction of the arm, under $\mathrm{c}$ arm visualization. The exposure of proximal humeral shaft was through making 'window' at delto-bicipital interval by preserving cephalic vein. A distal 'window' is put on proximal to the elbow crease anterior side. The musculocutaneous nerve and its sensory branch was explored and protected under retractor that is retracting the biceps muscle. By doing blunt dissection, brachialis muscle was not cut, but split. By using plate itself, a submuscular tunnel was made. Reduction was achieved by manual traction. A 9- to 12-hole $4.5 \mathrm{~mm}$ narrow dynamic compression plate was used for stabilization of fracture. Anterior surface of shaft humerus is where plate is usually put. Generally, minimum 3 bicortical screws (either locking or cortical screw) were inserted either side of fracture. ${ }^{4}$ Radial nerve was not explored.

\section{Posterior approach Open reduction and internal fixator (ORIF)}

All the patients were given lateral position on operating table with injured extremity hanging over bar making 90 degree flexion at elbow joint. Patient's body is stabilized by giving side supports on operating table. Intraoperative c-arm visualization is used. Tourniquets were not used and painting and draping was done in standard fashion. Posterior approach was used and skin incision was done over posterior aspect of arm which is distally extending in between lateral epicondyle and olecranon $2.5 \mathrm{~cm}$ distally to elbow joint. A variation of the standard posterior approach is the "triceps-sparing" or "para-tricipital" approach that provides good exposure to the distal humerus posteriorly, avoids injury to the triceps muscle with less risk of denervating a portion of the triceps or the anconeus, and therefore, may improve postoperative elbow function. Proximally tissues in between the long and lateral heads of triceps were dissected carefully to identify, isolate and separate radial nerve. ${ }^{5}$ Periosteum was isolated through use of periosteum elevator and proximal and distal humerus was aligned and fracture was reduced with the use of reduction clamps and plate was placed followed by fixing the plate with the use of locking and HCS screw.

\section{Results}

1. 50 patients having shaft humerus fracture managed with MIPPO anterior bridge plate and conventional ORIF by posterior platting were selected. ( 25 patients by MIPPO and another 25 by conventional ORIF)

2. The age of patients varied between 20-60 years, mean being 44 .

3. 29 of them were male while 21 were female.

4. Mode of injury was RTA in $68.7 \%$ of patients and fall down from height in $31.3 \%$.

5. Mean time of operation was 1 days from admission.

6. Average operating time was $120 \pm 10.5 \mathrm{~min}$ (109.5$130.5 \mathrm{~min}$ ) for conventional ORIF by single posterior plating while $100 \pm 10$ in $(90-110 \mathrm{~min})$ for MIPPO anterior plating.

7. Hospital stay varied from 7 days to 20 days, mean being 13 days.

8. Average time of callus formation was found to be 20.4 weeks. Concomitant injuries didn't hamper the bone healing but soft tissue recovery occurred in an uneventful manner.

9. 3 patients operated by standard posterior platting needed bone graft so autogenous bone graft was taken from ASIS.

10. Among 50 patients, 11 were having other associated injury like blunt chest, abdomen or head injury or other fracture including femur, radius ulna etc.

11. 7 patients ( $14 \%$ of all patients) were having primary radial nerve palsy

Operative duration for humerus fracture was calculated from skin incision to skin closure excluding some cases of poly-trauma where combined procedures were performed. The mean surgical time was approximately $120 \pm 10.5 \mathrm{~min}(109.5-130.5 \mathrm{~min})$ for conventional ORIF by single posterior plating while 100 \pm 10 in $(90-110 \mathrm{~min})$ for MIPPO anterior plating $(P<$ 0.05 , Wilcoxon's signed rank test). All the patients were regularly followed up and mean followed up time was 13 months (range from 06 to 20 months). One patient (2\%) in conventional ORIF group developed non-union at fracture site which require revision surgery with bone grafting. One patient (2\%) in conventional ORIF group was having post-operative infection which was covered with higher antibiotics and later on patient got recover. One patient $(2 \%)$ operated with posterior standard platting developed severe infection for which implant removal, revision platting plus bone grafting was done. 
In our study no patient have any complain of implant impingement. At the end in our study in final follow up, the Mayo Elbow Performance Score (MEPS) which is ranged from $95-100$ is used. $90 \%$ of total cases $(n=45)$ operated were showing excellent score, in which conventional ORIF posterior plating group had 20 patients and MIPPO plating group had 25 patients. 5 patients operated in which 2 were in ORIF posterior plating group and 3 patients were in MIPPO platting group showed a good score. All the patients can mobilise their elbow fully and the mean flexion at elbow joint is 153.5 degree and can do routine daily activity without any problems. In our study no limb shortening was observed.

\section{Functional outcomes \\ MAYO SCORE ${ }^{6}$}

\begin{tabular}{|l|l|c|c|}
\hline $\begin{array}{c}\text { Mayo Elbow } \\
\text { Performance } \\
\text { Score(MEPS) }\end{array}$ & Interpretation & $\begin{array}{c}\text { Patients operated } \\
\text { with single posterior } \\
\text { humerus plating }\end{array}$ & $\begin{array}{c}\text { Patients } \\
\text { operated with } \\
\text { MIPPO plating }\end{array}$ \\
\hline Score greater than 90 & Excellent & 20 & 25 \\
\hline Score 75-89 & good & 2 & 3 \\
\hline Score 60-74 & Fair & 0 & 0 \\
\hline Score below 60 & Poor & 0 & 0 \\
\hline
\end{tabular}

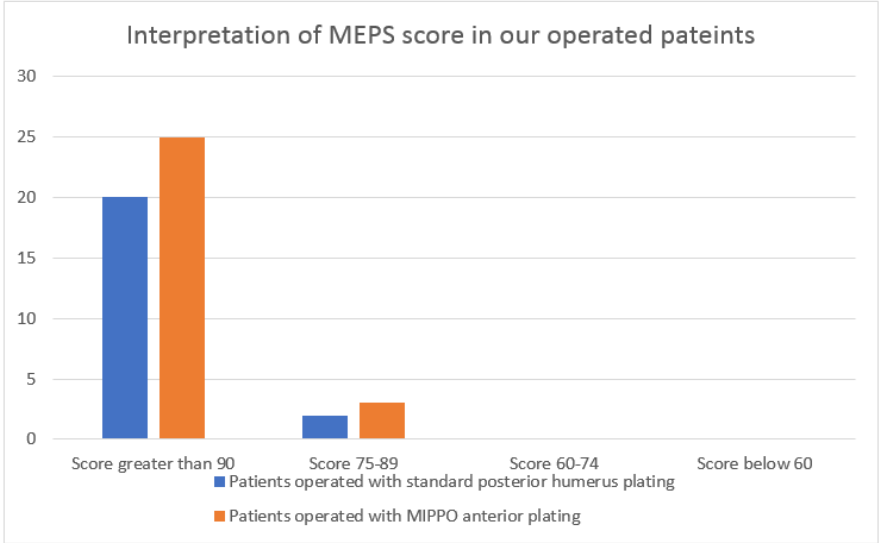

\section{UCLA score}

All patients get union within 7-8 months, except for one patient in the MIPPO group and two patients in the conventional ORIF group who failed to achieve primary union. Mean UCLA scores were 34.3 and 33.8 in the MIPPO and ORIF groups and mean Mayo elbow performance indices were 96.6 and $95 \%$ respectively. $(P>0.05)$.

\begin{tabular}{|c|c|c|}
\hline Parameters & $\begin{array}{c}\text { Conventional ORIF } \\
\text { posterior plating }\end{array}$ & $\begin{array}{c}\text { MIPPO anterior } \\
\text { plating }\end{array}$ \\
\hline Surgical mean time & $\begin{array}{c}120 \pm 10.5 \mathrm{~min}(109.5- \\
130.5 \mathrm{~min})\end{array}$ & $\begin{array}{c}100 \pm 10 \mathrm{in}(90-110 \\
\mathrm{min})\end{array}$ \\
\hline Average blood loss & $210 \pm 40 \mathrm{ml}$ & $150 \pm 40 \mathrm{ml}$ \\
\hline
\end{tabular}

\section{Discussion}

Platting has been the management of choice for shaft humerus fracture. Various complications like Healing complications, infections of situ and post op iatrogenic radial nerve palsy have been reported. Despite of that, platting provide primary contact healing without

\section{Radiographics outcomes}

Twenty-four of the $25(96 \%)$ MIPPO patients and twenty two of the $25(88 \%)$ ORIF patients achieved union. Average union times for the MIPPO and ORIF groups were 19.3 and 16.1 weeks respectively. Union rates and union times were almost equal in both methods $(P>0.05)$. All of type A fractures was united irrespective of conventional platting or MIPO. Two non unions ( 1 in occurred in ORIF group, while 1 occurred in MIPPO group) were observed among all the patients. In type $\mathrm{C}$ ractures, there was one non union (one case in ORIF ). Non union after MIPO was a comminuted fracture of distal shaft, with inappropriate fixation and poor stability and was done in hurry due to intra op anaesthetic complication. We presume that non unions after ORIF were caused by intra op mis-management of small, comminuted fragment of fracture. any callus formation so its always desirable over any other types of fracture healing. Plate osteosynthesis in case of comminuted humeral fracture requires surgical expertise and precise attention to crucial structures like neurovascular bundle, muscles and periosteum. Since ages, platting was done by opening the whole fracture 
site without damaging the neurovascular bundle and putting the plate by screws i.e conventional Open Reduction Internal Fixation (ORIF). For the treatment and management of humeral shaft fractures, minimally invasive percutaneous plate osteosynthesis (MIPPO) is a new developing surgical technique. ${ }^{7}$ One of the main advantages of MIPPO technique is that it preserves and protects the soft tissue and blood circulation of periosteum of fractured bone which boosts up fracture healing. In the MIPPO group of this study, most cases achieved primary osseous union. This was also comparable to the result of ORIF group. MIPPO is generally known to achieve better results for complex comminuted fractures of humeral shaft where as simple fractures are better managed with compression plating i.e. conventional ORIF. In this presented study, we assessed the rate of fracture union and time of union according to its classification, which were satisfactory in both methods. All simple fractures (type A) in MIPPO group are united. Accordingly, we assume that MIPPO may be a useful method of humerus fractures, irrespective of its fracture classification. To enhance fracture healing, usually bone graf is used in complex comminuted fractures. It may be beneficial for filling the unwanted gaps after plate fixation. Autogenous iliac cancellous bone grafts (AIBG) may give morbidity (up to $44 \%$ ) to the donor site like maralgia parasthetica in case of grafting from ASIS. In two patients in conventional ORIF, even though primary bone grafting was done, fracture went to non union. Consequently, it confirm that MIPPO gives high union rate without need of any bone graft. ${ }^{8}$ Mal-alignment or mal union is a common complication of MIPPO when it use in long bone fractures. However, in this presented study, not a single case of mal-alignment was observed in the MIPPO group, which counteract the previous results. On the other hand, a long use of C- Arm visualization is inevitable for MIPPO to have a satisfactory fracture alignment. This may reflect the relatively long radiation exposure time of MIPPO group in this study. The functional outcomes of shoulders and elbows were excellent according to Mayo and UCLA scoring methods in both study groups, which is consistent with previous reports on platting technique. Short surgical incision and intra operative time gives lower chances of infection. So, it concludes that even though MIPPO is surgically demanding, fracture hematoma is not disturbed in it therefore it gives better and fast union than conventional ORIF.

\section{Case Report}

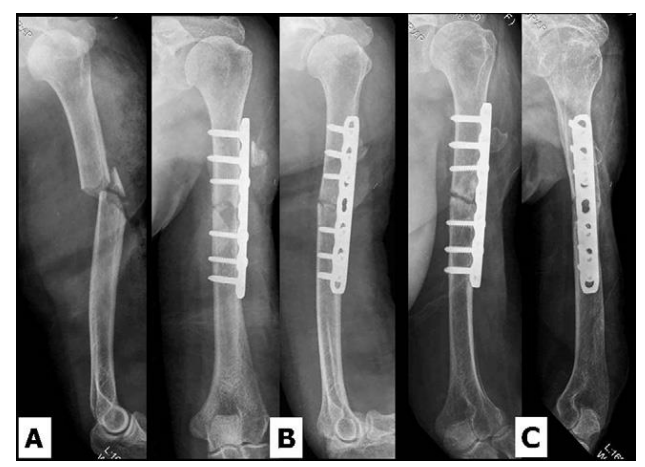

Fig. 1

A 60 years old female with proximal third fracture of the left humeral shaft (AO-OTA 12 B3) (A) injured after a RTA, was treated by open reduction and internal fixation (ORIF) by conventional posterior approach (B). After Six months post operatively, the fracture had not united (C).

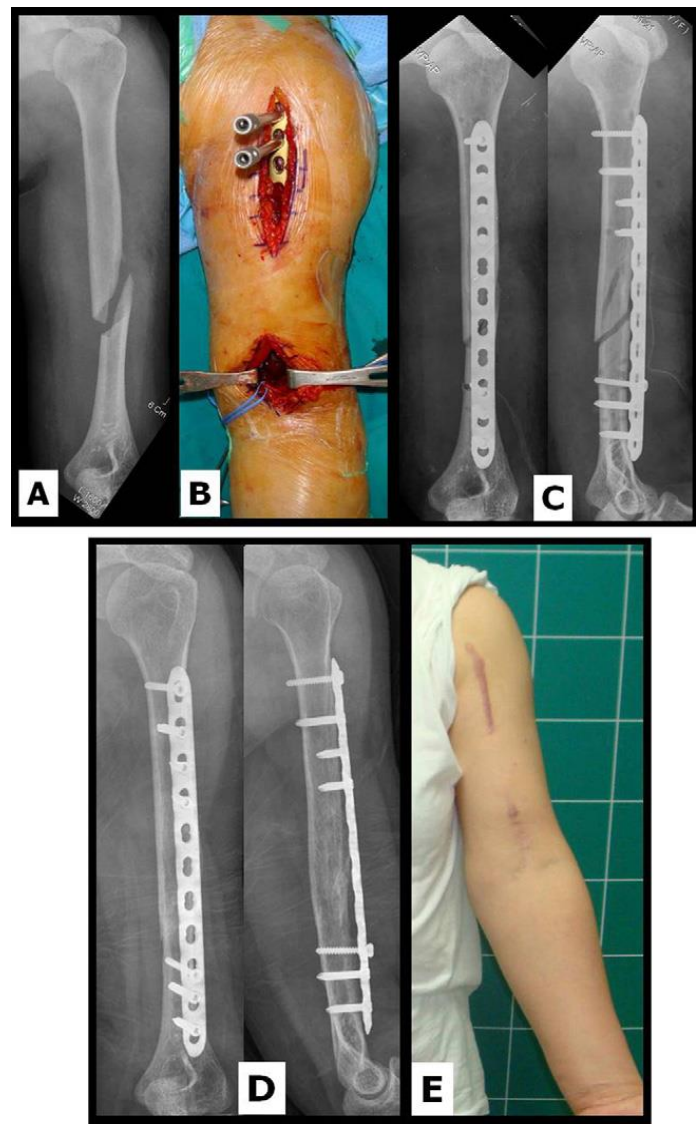

Fig. 2

A 47-year-old female with a fracture of the left humeral shaft (AO-OTA 12 A1) after Low velocity RTA (A). A plate was inserted through submuscular tunnel (B). Postoperative radiographs show an acceptable alignment (C). At 6 months Postop, radiographs shows a 
complete bony union (D) with a satisfactory function (E).

\section{References}

1. Paul Tornetta III MD, Charles Court-Brown MD, James D. Heckman MD, Michael McKee MD FRCS (C), Margaret M. McQueen MD, William Ricci MD, Rockwood and Green's Fractures in Adults, 2005; 1287 88

2. Ekholm R, Adami J, Tidermark J, et al. Fractures of the shaft of the humerus. An epidemiological study of 401 fractures. Journal of Bone Joint Surgery Br. 2006;88(11):1469-1473.

3. Marsh JL, Slongo TF, Agel J, et al. Fracture and dislocation classification compendium- 2007: Orthopaedic Trauma Association classification, database and outcomes committee. Journal of Orthopaedic Trauma. 2007;21(suppl 10):S1-S133.

4. Spagnolo R, Pace F, Bonalumi M. Minimally invasive plating osteosynthesis technique applied to humeral shaft fractures: The lateral approach. European Journal of Orthopaedic Surgery and Trauma tol. 2010;20:205-210.
5. Müller ME, Allgöwer M, Schneider R, et al. Manual of Internal Fixation. Techniques Recommended by the AOASIF Group. 3rd ed. Berlin: Springer-Verlag; 1991.

6. Morrey BF, An KN, Chao EYS. Functional evaluation of the elbow. In: Morrey BF, ed. The Elbow and its Disorders. 2nd ed. Philadelphia, PA: Saunders; 1993:8697.

7. Spagnolo R, Pace F, Bonalumi M. Minimally invasive plating osteosynthesis technique applied to humeral shaft fractures: The lateral approach. European Journal of Orthopaedic Surgery and Trauma tol. 2010;20:205-210.

8. Ji F, Tong D, Tang $\mathrm{H}$, et al. Minimally invasive percutaneous plate osteosynthesis (MIPPO) technique applied in the treatment of humeral shaft distal fractures through a lateral approach. International Orthopaedic. 2009;33(2):543-47.

How to cite this article: Anand K., Gol Abhishek, Mungalpara N., Usdadia S., Shiyal R. Operative Management of closed fracture shaft humerus: comparative study between MIPPO anterior bridge platting Vs conventional ORIF. IP Int J Orthop Rheumatol 2018;4(2):68-72. 